\title{
New tracking philosophy for birds
}

Peer-reviewed letter

In 1835 the English poet William Wordsworth wrote "Resplendent Wanderer! followed with glad eyes, Where'er her course; mysterious Bird! To whom, by wondering Fancy stirred", capturing both our fascination with bird movement and our helplessness to determine it. But things have since changed. Minute leg rings allow for banded individuals to be resighted by 

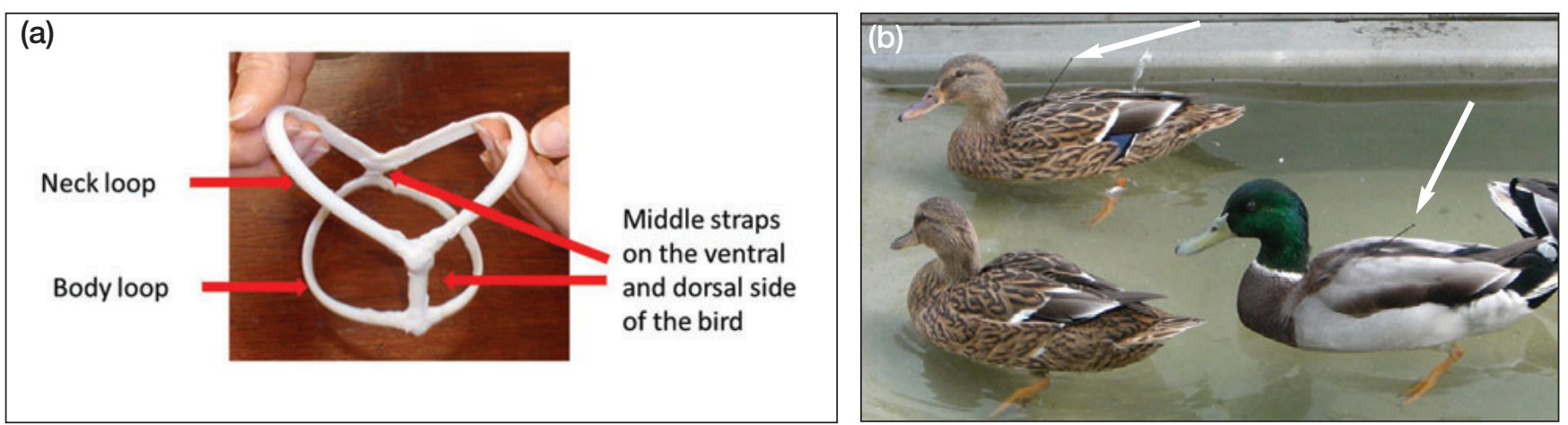

Figure 1. (a) As with other backpack harnesses, the Silastic harness is formed of two loops: one neck loop and one body loop joined together by middle straps. The V-shaped neck loop sits on the shoulders, passing under the sternum to avoid disrupting swallowing. The body loop sits around the main body, not too close to the moving limbs (ie wings and legs) with the wings going through the open spaces between the two loops. (b) Two mallards (Anas platyrhynchos) wearing the harness and carrying mock VHF transmitters. Note that no aspect of the harness is visible.

observers, thereby demonstrating avian dispersal capacities (Salomonsen 1956). But leg-ring sightings are subject to chance and only provide point locations in space with unknown trajectories between them. This problem was partially solved by "geolocation" (Wilson et al. 1992), in which miniature data loggers on leg rings record light intensity against Greenwich Mean Time, thereby allowing daily determination of latitude and longitude (eg Shaffer et al. 2006). As with leg rings, however, the miniature loggers must be recovered to retrieve the data. With the inception of radio (very high frequency [VHF]) telemetry, researchers were able to pinpoint the location of transmitter-equipped individuals without recapture; furthermore, when such transmitters (eg platform transmitter terminals [PTTs]) communicated with satellites, global coverage of bird movements became available (eg Jouventin and Weimerskirch 1990). Such devices are unlikely to ever work on leg rings, however, being too large and with ventral positioning hindering tag-satellite communication. Ideally, these devices should be positioned dorsally, although physical attachment in this position is challenging. Methods to tape (Wilson and Wilson 1989) and glue (Raim 1978; Sykes et al. 1990) tags to dorsal feathers work well until the feathers are molted, limiting deployment duration to weeks (Warnock and
Warnock 1993), and harnesses have an inconsistent performance history (Rappole and Tipton 1991), with some reports pointing to behavioral anomalies (Perry 1981), feather wear or skin abrasion (Buehler et al. 1995), and even mortality (Peniche et al. 2011).

The problem with harnesses may lie in their conception as being able to withstand all possible environmental conditions as well as unwelcome attention from the bird itself, which explains why Teflon is popular despite its mixed success (Steenhof et al. 2006). We tried a fundamentally different approach, fashioning a harness from a soft, elastic, hypoallergenic, silicone-based compound called Silastic (Dow Corning Corporation, Midland, MI) that is designed to sit directly on the skin under the plumage (Figure 1). The idea behind this was that if the attachment system was imperceptible (both visually and by touch) and protected by the birds' feathers, it would be subject neither to external conditions nor to the attention of the wearer. In addition, Silastic's variable elasticity should be able to accommodate seasonal mass changes in outfitted birds. Our work has proceeded carefully; with trials ranging from days to months, we examined potential behavioral anomalies and possible plumage and skin changes on example species of passerines, gulls, ducks, and corvids, with no deleterious effects observed (WebTable 1).
Nineteen adult jackdaws (Coloeus monedula) wore VHF and PTT tags for a maximum of 5 months in captivity before being successfully released in the wild.

Other researchers are also experimenting with a similar approach. Two teams have recently reported recovering tags attached with elastic harnesses from northern wheatears (Oenanthe oenanthe; Bairlein et al. 2012) and hoopoes (Upupa epops epops; Bächler et al. 2010) after yearlong deployments on wild birds migrating across hemispheres and trans-equatorially, respectively. Such long periods and arduous migrations are a severe test for the attachment protocol, and the results appear promising.

Ultimately, the ability to resolve normal bird movements over long time periods depends on both an appropriate attachment mechanism and minimal tag size, while still maintaining tag contact with a satellite. Future success in this approach has been facilitated by the confirmation that a VHF receiver, dedicated to detection of animal-borne VHF transmitters, will be carried in 2014 by the low-orbiting International Space Station, which is supported by the European Space Agency and the German Air and Space Agency (Pennisi 2011). This system relies on modern CDMA (code division multiple access) communication technology in miniaturized electronic circuits and thus will not only help 
to shrink animal tag size but also allow for an expansion in onboard nano-sensing technology. Currently, the ICARUS (International Cooperation for Animal Research Using Space) initiative plans to have $5-\mathrm{g}$ Global Positioning System logging tags and 1-g communication-only tags ready for experiments in 2015.

With technological advances in harnesses and solar-powered transmitters, as well as remote access to satellites, wildlife biologists should be able to track individual birds for years, revealing information on, for instance, where birds perish (eg Burnham and Newton 2011), addressing both conservation and pure research issues. Empowered with such science, we may see a day when Wordsworth followers will appreciate that there is no more mystery in bird paths, leaving them to lyricize about remarkable avian traveling feats instead.

Sylvie P Vandenabeele ${ }^{1 *}$, Rory $\mathrm{P}$ Wilson ${ }^{1}$, and Martin Wikelski ${ }^{2}$ ${ }^{1}$ Swansea Moving Animal Research Team, Biosciences, College of Science, Swansea University, Swansea, UK *(574139@swansea.ac.uk); ${ }^{2}$ Max Planck Institute for Ornithology, Vogelwarte Radolfzell, Radolfzell, Germany

Bächler E, Hahn S, Schaub M, et al. 2010. Year-round tracking of small transSaharan migrants using light-level geolocators. PLOS ONE 5: 4.

Bairlein F, Norris DR, Nagel R, et al. 2012. Cross-hemisphere migration of a $25 \mathrm{~g}$ songbird. Biol Lett 8: 505-07.

Buehler DA, Fraser JD, Fuller MR, et al. 1995. Captive and field-tested radio transmitter attachments for bald eagles. J Field Ornithol 66: 173-80.

Burnham KK and Newton I. 2011. Seasonal movements of gyrfalcons Falco rusticolus include extensive periods at sea. Ibis 153: 468-84.

Jouventin P and Weimerskirch H. 1990. Satellite tracking of wandering albatrosses. Nature 343: 746-48.

Peniche G, Vaughan-Higgins R, Carter I, et al. 2011. Long-term health effects of harness-mounted radio transmitters in red kites (Milvus milvus) in England. Veterinary Record 169: 311.

Pennisi E. 2011. Global tracking of small animals gains momentum. Science 334: 1042.

Perry MC. 1981. Abnormal behavior of canvasbacks equipped with radio transmitters. J Wildlife Manag 45: 786-89.

Raim A. 1978. A radio transmitter attachment for small passerine birds. Bird Banding 49: 326-32.

Rappole JH and Tipton AR. 1991. New harness design for attachment of radio transmitters to small passerines. J Field Ornithol 62: 335-37.

Salomonsen F. 1956. The Greenland birdbanding system. Arctic 9: 258-64.

Shaffer S, Tremblay Y, Weimerskirch H, et al. 2006. Migratory shearwaters integrate oceanic resources across the Pacific Ocean in an endless summer. $P$ Natl Acad Sci USA 103: 12799-12802.

Steenhof K, Bates KK, Fuller MR, et al. 2006. Effects of radiomarking on prairie falcons: attachment failures provide insights about survival. Wildlife Soc Bull 34: 116-26.

Sykes PWJ, Carpenter JW, Holzman S, and Geissler PH. 1990. Evaluation of three miniature radio transmitter attachment methods for small passerines. Wildlife Soc Bull 18: 41-48.

Warnock N and Warnock S. 1993. Attachment of radio-transmitters to sandpipers: review and methods. Wader Study Group Bull 70: 28-30.

Wilson RP, Ducamp JJ, Rees WG, et al. 1992. Estimation of location: global coverage using light intensity. In: Priede IM and Swift SM (Eds). Wildlife telemetry: remote monitoring and tracking of animals. Chichester, UK: Ellis Horward.

Wilson RP and Wilson M-PTJ. 1989. Tape: a package-attachment technique for penguins. Wildlife Soc Bull 17: 77-79.

doi:10.1890/13.WB.002 